Sādhanā Vol. 37, Part 5, October 2012, pp. 539-556. (C) Indian Academy of Sciences

\title{
Fuzzy clustering of mechanisms
}

\author{
AMITABHA GHOSH $^{1}$, DILIP KUMAR PRATIHAR ${ }^{2, *}$, \\ M V V AMARNATH ${ }^{2}$, GUENTER DITTRICH ${ }^{3}$ and \\ JORG MUELLER ${ }^{3}$
}

\author{
${ }^{1}$ Bengal Engineering and Science University, Howrah, 711103, India \\ ${ }^{2}$ Department of Mechanical Engineering, Indian Institute of Technology Kharagpur, \\ Kharagpur, 721302, India \\ ${ }^{3}$ Institut fuer Getriebetechnik und Maschinendynamik, RWTH Aachen, 52062 \\ Aachen, Germany \\ e-mail: dkpra@mech.iitkgp.ernet.in
}

MS received 27 February 2011; revised 9 May 2012; accepted 28 June 2012

\begin{abstract}
During the course of development of Mechanical Engineering, a large number of mechanisms (that is, linkages to perform various types of tasks) have been conceived and developed. Quite a few atlases and catalogues were prepared by the designers of machines and mechanical systems. However, often it is felt that a clustering technique for handling the list of large number of mechanisms can be very useful, if it is developed based on a scientific principle. In this paper, it has been shown that the concept of fuzzy sets can be conveniently used for this purpose, if an adequate number of properly chosen attributes (also called characteristics) are identified. Using two clustering techniques, the mechanisms have been classified in the present work and in future, it may be extended to develop an expert system, which can automate type synthesis phase of mechanical design. To the best of the authors' knowledge, this type of clustering of mechanisms has not been attempted before. Thus, this is the first attempt to cluster the mechanisms based on some quantitative measures. It may help the engineers to carry out type synthesis of the mechanisms.
\end{abstract}

Keywords. Clustering; mechanisms; fuzzy C-means algorithm; entropy-based algorithm; genetic algorithm; self-organizing maps.

\section{Introduction}

While designing a mechanical system to serve a specific purpose, one of the important tasks faced by the designers is to understand its working principle and select an appropriate mechanism for it. Designers use the available atlases and catalogues of mechanisms, and also their experiences to select a suitable one depending on the application. This task of selecting a suitable

*For correspondence 
mechanism can be facilitated, if the mechanisms can be clustered based on their functional characteristics. A simple scheme of clustering may not be always very convenient, unless physical significance can be associated with the clustering elements and the description is amenable to be handled in a quantitative manner. Development of a scheme with a quantitative sense attached to the clusters may facilitate the process and can even make it possible to automate the same based on a procedure of optimization.

Since the publication of the seminal paper by Zadeh (1965), the concept of fuzzy sets has been successfully employed in many areas, and clustering is one of those. Group Technology has very successfully used the concept of fuzzy sets for part family formation (Wang \& Li 1991), and it can also be used for clustering and classification of mechanisms. However, a very essential thing is to identify proper attributes (also called characteristics), which can be used as the features supported by the mechanisms.

Once the feasibility of using the concept of fuzzy clustering of mechanisms is demonstrated, this idea may be extended to develop a scheme of multi-stage clustering. After the groups are formed at the primary stage, the groups (especially those with a large number of members) can be subjected to further grouping based on the sets of more specialized attributes (specific to individual cluster). To the best of the authors' knowledge, no study has been reported on fuzzy clustering of mechanisms.

The objective of this paper has been restricted to only demonstrate the feasibility of using the concept of fuzzy clustering of mechanisms. Only 80 mechanisms have been used as an example for this purpose. In the present work, no attempt has been made to initiate the scheme of multistage clustering. Only a single-stage clustering using a set of primary attributes has been taken up.

The remaining part of this paper has been organized as follows: Section 2 introduces the role of fuzzy sets in formation of the clusters of mechanisms. The working principles of two fuzzy clustering algorithms have been explained in section 3. Results are stated and discussed in section 4. Some concluding remarks are made in section 5. The scopes for future study have been discussed in section 6 .

\section{Role of fuzzy sets in clustering of mechanisms}

Mechanisms may be clustered based on the concept of similarity, which can be determined with the help of their attributes. There exist a large number of mechanisms to perform various tasks, and some of those might be overlapping in terms of their attributes. A mechanism can be described with reference to various attributes using the concept of membership (that is, degree of belongingness) utilized in the fuzzy sets (Zadeh 1965). It is also understood that the attributes might have to be classified as the primary, secondary, and so on, to get the best results. The primary attributes refer to the basic functional aspects, whereas the secondary attributes deal with the specialized and specific details relevant to a particular cluster of mechanisms only. Thus, the former helps to group the mechanisms into a few clusters and the latter sub-divides a particular cluster into a number of sub-clusters.

In this paper, only a single-stage clustering has been taken up to show the feasibility and explain the procedure. One of the most important matters in fuzzy clustering of mechanisms is the choice of an adequate number of suitable attributes. It is also true that no unique list of suitable attributes is possible to make. The only way to resolve the issue is to gradually approach a set of suitable attributes by a trial and error method. Once the clusters obtained through an iterative method are found to be physically significant, the set can be considered to be acceptable. 
Each mechanism is assigned a membership value (lying between 0.0 and 1.0) with respect to each of its attributes. If the mechanism satisfies the attribute perfectly, the membership value is made equal to 1.0. On the other hand, if it does not satisfy the attribute at all, then the membership value becomes equal to 0.0. Appendix A shows a list of 80 mechanisms considered for the present study. The membership values of these mechanisms with respect to twenty different attributes have been decided by the first, fourth and fifth authors of this paper, after consulting a group of experts working in the field of mechanisms, as shown in Appendix B. Therefore, each data entry shown in Appendix B has been made after consulting a group of experts working in the field of mechanisms.

\section{Clustering algorithms}

There exist a large number of fuzzy clustering algorithms, namely fuzzy ISODATA, fuzzy cmeans, fuzzy k-nearest neighbourhood, potential-based clustering, entropy-based clustering, and others (Pratihar 2008). Out of these algorithms, Fuzzy C-Means (FCM) algorithm has received much attention due to its reasonably good performance and simplicity. Moreover, Entropy-based Fuzzy Clustering (EFC) algorithm is seen to be a more flexible algorithm compared to the FCM algorithm (Pratihar 2008). It is to be noted that the performance of a clustering algorithm is data dependent. Therefore, it may be difficult to select an appropriate clustering algorithm beforehand to be used to tackle a set of data points. The working principles of two clustering techniques, namely EFC and FCM used in the present study, are explained below, in brief.

\subsection{Entropy-based fuzzy clustering algorithm}

Entropy-based fuzzy clustering (EFC) is an iterative algorithm, in which entropy values of the data points are determined first, and then the data point having the minimum entropy value is selected as the cluster center (Yao et al 2000). The remaining data points are clustered according to the threshold value of similarity. The data points, which cannot be put inside any of the clusters, are termed as outliers. The principle of EFC is discussed below.

Let $N$ data points in $M$-dimensional [T] hyperspace be considered, where each data point $X_{i}$ ( $\mathrm{i}=1,2,3, \ldots, N)$ is denoted by a set of $M$ values (that is, $X_{i 1}, X_{i 2}, X_{i 3}, \ldots \ldots X_{i M}$ ). Therefore, the data set can be represented by an $N \times M$ matrix. The Euclidean distance between any two data points (for example, $i$ and $j$ ) is calculated as follows:

$$
D_{i j}=\sqrt{\sum_{k=1}^{M}\left(X_{i k}-X_{j k}\right)^{2}} .
$$

Now, similarity between these two points can be determined like the following:

$$
S_{i j}=e^{-\alpha D_{i j}}
$$

where $\alpha$ is a constant, which is determined as follows:

$$
\alpha=-\frac{\ln 0.5}{\bar{D}} .
$$


Here, $\bar{D}$ is the mean distance calculated as follows:

$$
\bar{D}=\frac{1}{{ }^{N} C_{2}} \sum_{i=1}^{N} \sum_{j=1}^{N, j<i} D_{i j}
$$

The entropy of each data point $E_{i}$ can be calculated with respect to other data points as given below.

$$
E_{i}=-\sum_{j \in X}^{j \neq i}\left(S_{i j} \log _{2} S_{i j}\right)+\left(1-S_{i j}\right) \log _{2}\left(1-S_{i j}\right) .
$$

The EFC algorithm consists of the following steps:

Step 1: Calculate $E_{i}(\mathrm{i}=1,2,3, \ldots, \mathrm{N})$ for each $X_{i}$ lying in $[T]$ hyperspace.

Step 2: Determine minimum $E_{i}$ and declare $X_{i, \operatorname{Min}}$ as the cluster center.

Step 3: Put $X_{i, \text { Min }}$ and the data points having similarity with $X_{i, \text { Min }}$ greater than $\beta$ (threshold value for similarity) into a cluster and remove them from $[T]$.

Step 4: Check whether [T] hyperspace is empty. If yes, terminate the program, else go to Step 2.

In this algorithm, $E_{i}$ is calculated in such a way that a data point that is far away from the remaining ones may also be selected as a cluster center. To prevent such an odd situation, a threshold value of $\gamma$ (in $\%$ ) is used. If the number of data points of a cluster becomes either greater than or equal to $\frac{\gamma N}{100}$, we declare it a valid one. Otherwise, these data points will be treated as the outliers.

\subsection{Fuzzy C-means algorithm}

Fuzzy C-Means (FCM) algorithm is one of the most popular fuzzy clustering techniques, in which the data points have their membership values with the cluster centers, which will be updated iteratively (Dunn 1973; Bezdek 1973). Let us consider $M$-dimensional $N$ data points denoted by $X_{i}(i=1,2, \ldots, N)$, which are to be clustered. The FCM algorithm consists of the following steps:

Step 1: Assume the number of clusters to be made, that is, $c$, where $2 \leq c \leq N$.

Step 2: Select an appropriate level of cluster fuzziness $f>1$.

Step 3: Initialize the $N \times c$ sized membership matrix [U], at random, such that $U_{\mathrm{ij}} \in[0,1]$ and $\sum_{j=1}^{C} U_{i j}=1.0$, for each $i$.

Step 4: Determine the $k^{\text {th }}$ dimension of $j^{\text {th }}$ cluster center, that is, $C C_{\mathrm{jk}}$ using the expression given below.

$$
C C_{j k}=\frac{\sum_{i=1}^{N} U_{i j}^{f} x_{i k}}{\sum_{i=1}^{N} U_{i j}^{f}}
$$

Step 5: Calculate the Euclidean distance between $i^{\text {th }}$ data point and $j^{\text {th }}$ cluster center like the following.

$$
D_{i j}=\left\|\left(x_{i}-C C_{j}\right)\right\|
$$


Step 6: Update fuzzy membership matrix [ $U$ ] according to $D_{\mathrm{ij}}$. If $D_{\mathrm{ij}}>0$, then

$$
U_{i j}=\frac{1}{\sum_{k=1}^{c}\left(\frac{D_{i j}}{D_{i k}}\right)^{\frac{2}{f-1}}} .
$$

If $D_{\mathrm{ij}}=0$, then the data point will coincide with $j^{\text {th }}$ cluster center $C C_{j}$ and it will have the full membership value, that is, $U_{\mathrm{ij}}=1.0$.

Step 7: Repeat Steps 4 through 6, until the changes in $[U]$ become less than some pre-specified values.

The validity of clusters is to be checked as discussed above using a parameter $\gamma$.

\subsection{GA-fitness calculation}

The performances of the said clustering algorithms depend on a number of parameters. However, the performance of a clustering algorithm cannot be mathematically expressed as the function of its parameters. Therefore, it might be difficult to use a local optimizer like steepest descent method to carry out the necessary optimization. A powerful global optimizer, namely binarycoded Genetic Algorithm (GA) (Holland 1975; Goldberg 1989) has been used, in this study. Ten bits are assigned to represent each real variable. In order to improve the performance of EFC algorithm, three real variables, namely $\alpha, \beta$ and $\gamma$ are optimized using the GA, whereas two real variables, such as $f$ and $\gamma$ have been varied to optimize the performance of the FCM algorithm. The aim of the present study is to obtain the set of optimal (in terms of both compactness as well as distinctness) clusters. Moreover, the number of outliers should be as minimum as possible. The compactness of a cluster is determined by calculating average Euclidean distance of the members of a cluster from its center. The lower the value of average Euclidean distance, the more compact will be the cluster. The distinctness of the developed clusters is determined by calculating the average Euclidean distance of the cluster centers. The clusters are said to be more distinct, if the average Euclidean distance among their centers becomes more. The above problem has been formulated as a maximization problem, where the fitness $f^{\prime}$ of the GA-string is determined as given below.

$$
f^{\prime}=\frac{1}{1+\frac{1}{c} \sum_{k=1}^{c}\left(\frac{1}{n_{k}} \sum_{i=1}^{n_{k}} \sqrt{\sum_{j=1}^{M}\left(x_{i j}-v_{k j}\right)^{2}}\right)}+\frac{1}{{ }^{c} C_{2}} \sum_{i=1}^{c} \sum_{k=1, k \neq i}^{c} \sqrt{\sum_{j=1}^{M}\left(v_{i j}-v_{k j}\right)^{2}}-O^{\prime},
$$

where $c$ represents the number of clusters, $n_{k}$ indicates the number of points in $k^{\text {th }}$ cluster, $M$ denotes the dimension of the data point, $x_{i j}$ represents the $j^{\text {th }}$ dimension of $i^{\text {th }}$ data point, $v_{k j}$ indicates the $j^{\text {th }}$ dimension of $k^{\text {th }}$ cluster center and $O^{\prime}$ denotes the number of outliers.

The GA starts with a population of binary strings generated at random. As the initial strings are created at random, there is no guarantee that all of these will be equally good in terms of their fitness values, as calculated using equation (9). An operator named reproduction is used first to create a matting pool consisting of good strings hopefully. These solutions are then further modified using the operators, namely crossover and mutation. There will be an exchange of properties between two parents in crossover and consequently, children strings will be created. Mutation brings a local change to the strings to avoid the problem of local minimum (if any). It completes one generation of a GA cycle and this procedure is repeated, till it reaches the 
termination criterion. Interested readers may refer to (Pratihar 2008; Holland 1975; Goldberg 1989) for a detailed description of the algorithm.

\section{Results and discussion}

Results of the above two algorithms used to cluster the mechanisms are discussed below.

\subsection{Entropy-based fuzzy clustering}

The performance of the entropy-based fuzzy clustering (EFC) algorithm depends on the parameters like $\alpha, \beta$ and $\gamma$. An attempt has been made, in the present study, to cluster the above data in an optimal sense. As the performance of the GA is dependent on its parameters, namely probability of crossover $p_{c}$, probability of mutation $p_{m}$, population size $Y$ and maximum number of generations $G_{\max }$, a parametric study is conducted to determine the set of optimal GAparameters, in which one parameter has been varied at a time, keeping the others unaltered (Pratihar 2008). The following GA-parameters are found to yield the best results:

$\begin{array}{ll}\text { Crossover probability } p_{c} & =1.0 \\ \text { Mutation probability } p_{m} & =0.03 \\ \text { Population size } Y & =100 \\ \text { Maximum number of generations } G_{\max } & =100\end{array}$

During the optimization, the parameters, namely $\alpha, \beta$ and $\gamma$ are varied in the ranges of (0.6, $2.0),(0.35,0.80)$ and $(0.03,0.1)$, respectively. Ten bits are utilized to represent each of the said variables and consequently, the GA-string is considered to be 30-bits long. The optimal values of $\alpha, \beta$ and $\gamma$ are found to be equal to $0.6,0.35$ and 0.035 , respectively. It is interesting to observe that the optimal values of $\alpha$ and $\beta$ are found to match with the lower bound of their respective ranges. However, the above set of optimal values for $\alpha, \beta$ and $\gamma$ is not unique, as the GA is found to yield the optimized clusters of mechanisms with multiple sets of the said clustering parameters. Seven clusters are obtained containing 13, 16, 14, 15, 14, 5 and 3 mechanisms, respectively. Table 1 shows the cluster centers and carries information of the content of each cluster. The value of average Euclidean distance among the cluster centers representing distinctness and that of the members of the clusters from their respective centers indicating compactness have been found to be equal to 2.2660 and 1.1765 , respectively.

Table 1. Clusters of mechanisms obtained by EFC algorithm.

\begin{tabular}{lccl}
\hline $\begin{array}{l}\text { Cluster } \\
\text { No. }\end{array}$ & $\begin{array}{c}\text { No. of mechanisms } \\
\text { in the cluster }\end{array}$ & $\begin{array}{c}\text { Cluster center-Sl. } \\
\text { No. of mechanism }\end{array}$ & \multicolumn{1}{c}{ Mechanisms present in the cluster } \\
\hline 1 & 13 & 80 & $20,21,22,23,24,34,42,44,46,51,64,77,80$ \\
2 & 16 & 67 & $2,3,10,12,18,30,37,39,47,48,49,59,61,65,66,67$ \\
3 & 14 & 58 & $9,11,14,15,16,17,19,31,32,40,55,56,58,60$ \\
4 & 15 & 36 & $1,5,6,7,8,13,25,29,35,36,41,45,62,63,75$ \\
5 & 14 & 72 & $28,52,53,54,57,68,69,70,71,72,74,76,78,79$ \\
6 & 5 & 27 & $26,27,38,50,73$ \\
7 & 3 & 4 & $4,33,43$ \\
\hline
\end{tabular}


In order to visualize the obtained clusters, higher dimensional data of the mechanisms (shown in Appendix B) have been mapped into 2-D using a tool named Self-Organizing Map (SOM) (Kohonen 1995), as we cannot visualize more than 3-D space. A SOM is a special type of neural network, which works based on the principle of unsupervised learning. It is a topology-preserving technique for dimensionality reduction and an efficient tool for visualization. Interested readers may refer to Pratihar (2008) for a detailed description of this algorithm. Figure 1 displays a set of seven clusters of mechanisms obtained using this algorithm. It is interesting to note that this algorithm is able to yield a set of distinct clusters. Thus, eighty mechanisms considered in this study have been clustered into seven groups based on their similarities among them. As the SOM is a topology preserving mapping tool, it uses information related to not only the distance between two points but also their relative positions, that is, topology. In order to map higher dimensional data into a lower dimension, the distance information is utilized with the help of a scaling factor.

In the present study, 20 attributes of the mechanisms have been considered and consequently, 21-D data have been mapped into 2-D approximately for visualization, as more than 3-D cannot be visualized by the human beings. It is important to note that two axes of figure 1 represent dimensionless numbers. Moreover, the numerical values shown along the $\mathrm{X}$ and $\mathrm{Y}$ axes are dependent on the randomly chosen coordinate of the first point during the mapping. Thus, with another set of coordinate values for the first point, the numerical values along the $\mathrm{X}$ and $\mathrm{Y}$ axes could be different. However, the topology of the mapped points in 2-D will remain the same. It is important to mention that this figure might help an engineer to get a visual impression of various clusters present among the mechanisms. However, a designer or practical engineer will be more interested to know multidimensional attributes of the mechanisms rather than their 2-D information.

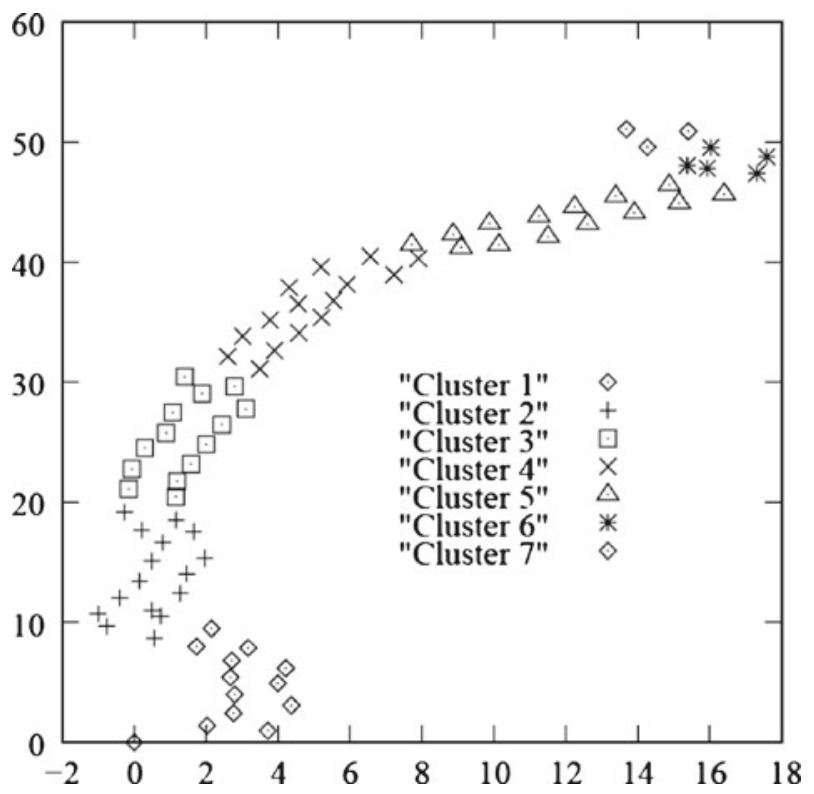

Figure 1. Clusters of mechanisms obtained by EFC algorithm. 


\subsection{Fuzzy C-means clustering}

The performance of the fuzzy C-means clustering (FCM) algorithm is dependent on the parameters like level of fuzziness $f$, number of clusters and threshold value used to check the validity of the clusters (that is, $\gamma$ ). As seven clusters are obtained using the EFC algorithm, the number of clusters to be made is kept equal to seven only, in the FCM algorithm. The optimal values of $f$ and $\gamma$ have been determined using a GA. The following GA-parameters obtained through a careful parametric study are seen to give the best results:

$\begin{array}{ll}\text { Crossover probability } p_{c} & =1.0 \\ \text { Mutation probability } p_{m} & =0.05 \\ \text { Population size } Y & =100 \\ \text { Maximum number of generations } G_{\max } & =150 .\end{array}$

The parameters, such as $f$ and $\gamma$ are varied in the ranges of $(1.1,8.0)$ and $(0.02,0.1)$, respectively, during optimization carried out using the GA. Ten bits are assigned to represent each of the above two variables. Thus, the GA-string is assumed to be 20-bits long. The optimal values of $f$ and $\gamma$ are seen to be equal to 1.1 and 0.05 , respectively. It is important to mention that the GA is able to provide multiple sets of $f$ and $\gamma$, corresponding to the same optimal fitness value, and the above reported set (that is, $f=1.1$ and $\gamma=0.05$ ) is one of them. Table 2 shows the mechanisms contained in seven clusters, as obtained by the FCM algorithm. It is important to mention that the cluster centers obtained by the FCM algorithm are hypothetical in nature. They may not exactly match with any one of the eighty mechanisms considered in the present study. The values of distinctness and compactness are obtained as 1.7267 and 0.7704 , respectively.

Higher dimensional clusters of mechanisms have been mapped into 2-D using the SOM (Kohonen 1995) for visualization. The set of clusters obtained utilizing the FCM algorithm is shown in figure 2, where some of the data points belonging to different clusters are found to overlap one another. The clusters yielded by this algorithm are found to be less distinct compared to those obtained by the EFC algorithm. It is important to mention that two axes of figure 2 carry dimension-less numbers, the reasons for which have been explained above. This figure could provide visual display of the clusters only. However, a practical engineer will be more interested in multi-dimensional space of mechanisms' attributes rather than their 2-D information, which can also be obtained from the in-built look-up table of the SOM.

Table 2. Clusters of mechanisms obtained by FCM algorithm.

\begin{tabular}{lccl}
\hline $\begin{array}{l}\text { Cluster } \\
\text { No. }\end{array}$ & $\begin{array}{c}\text { No. of mechanisms } \\
\text { in the cluster }\end{array}$ & $\begin{array}{c}\text { Cluster center-Sl. } \\
\text { No. of mechanism }\end{array}$ & \multicolumn{1}{c}{ Mechanisms present in the cluster } \\
\hline 1 & 18 & - & $\begin{array}{l}20,21,22,23,24,25,26,27,34,38,42,44,46, \\
\end{array}$ \\
2 & 12 & - & $50,51,64,73,77,80$ \\
3 & 14 & - & $2,3,4,12,18,25,30,43,47,48,49,65$ \\
4 & 17 & - & $9,11,14,15,16,17,19,31,32,40,55,56,58,60$ \\
5 & 8 & - & $1,5,6,7,8,13,28,29,35,36,37,41,45,59,62,63,75$ \\
6 & 6 & - & $73,52,53,54,57,68,69,79$ \\
7 & 5 & - & $10,39,61,66,67$ \\
\hline
\end{tabular}




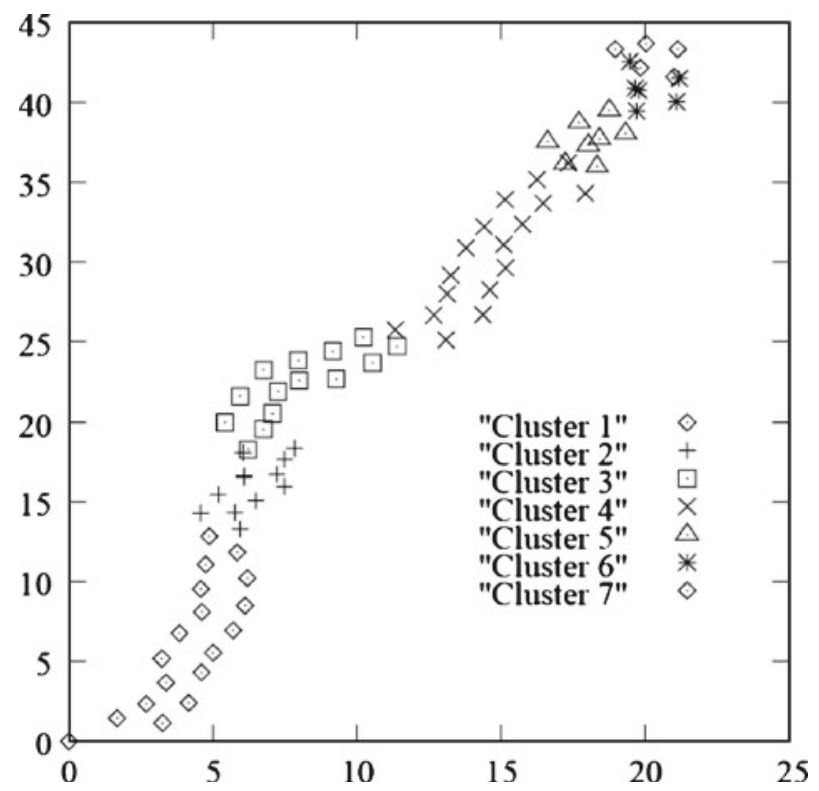

Figure 2. Clusters of mechanisms obtained by FCM algorithm.

A close watch on tables 1 and 2 reveals that the first four clusters (out of a total of seven clusters) generated by both the algorithms are almost similar (but not exactly the same). It is also interesting to note that the fifth cluster of table 1 has been approximately divided into two clusters (that is, $5^{\text {th }}$ and $6^{\text {th }}$ ), as shown in table 2 . However, the seventh cluster obtained by the above two algorithms are found to be different. As the performance of a clustering technique depends on its inherent parameters, it may not be always possible to obtain the same set of clusters for a particular set of data points using two different algorithms. It is also important to note that out of seven clusters; only five are found to be more distinct and prominent, as discussed below.

The first cluster contains some mechanisms having finite and limited input and output motions, which are suitable for guiding a rigid body from one location to another. The second cluster consists of a few mechanisms suitable for transforming angular motion to rectilinear motion and vice-versa, generating a straight-line motion. The third cluster is formed by the mechanisms suitable for taking continuous rotary input and generating fluctuating and intermittent motion. Moreover, the mechanisms suitable for converting continuous motion into oscillating motion and vice-versa are grouped into the fourth cluster. The fifth cluster consists of a few mechanisms capable of taking continuous rotary input and producing rotary/linear output; and suitable for controlling/adjusting input-output relationship. It is interesting to observe that both the clustering algorithms are able to yield almost the similar type of clusters (but not exactly the same). This difference has come due to the inherent properties of these two clustering algorithms. Several mechanisms have been designed and developed to serve a variety of purposes. Both the above clustering algorithms are able to cluster the mechanisms based on the type of tasks they perform. It is to be noted that the nature of input(s) and output(s) of a mechanism, and their transformation mainly depend on the type of its applications. Thus, it helps to device a quantifiable procedure for type synthesis of mechanisms, which may be helpful to the engineers. It may 
also assist a designer to select an appropriate mechanism for performing a particular task. Based on this study of clustering of mechanisms, an expert system may also be developed in future, which can help even a practical engineer to decide a suitable mechanism in order to solve a problem.

Clustering is done based on similarity and consequently, the similar mechanisms with respect to the attributes have come under the same cluster and dissimilar mechanisms have found their places in different clusters. The clusters obtained by the FCM algorithm are seen to be more compact compared to those generated by the EFC algorithm. It has happened due to the fact that in FCM algorithm, the cluster centers are initially selected at random and then updated iteratively. Thus, this algorithm tries to yield more and more compact clusters iteratively by updating their centers. On the other hand, the clusters determined by the EFC algorithm are seen to be more distinct compared to those obtained by the FCM algorithm. As expected, the clusters yielded by the FCM algorithm may also overlap each other (refer to figure 2). It is so, due to the reason that in FCM algorithm, the cluster centers are evolved artificially in an iterative way, which may not match with the data points used for the clustering. Thus, there is a less control on the selection of cluster centers and consequently, there might be an overlapping of the clusters. On the other hand, the cluster centers are selected from the set of data points to be clustered in the EFC algorithm and thus, the clusters have become more distinct (refer to figure 1). To summarize, although a more distinct set of clusters has been yielded by the EFC algorithm compared to the FCM algorithm, the latter has given a more compact set of clusters than the former. The reasons behind obtaining these outcomes have been explained above. However, the clusters should ideally be distinct as well as compact too.

It is important to mention that the performances of the clustering algorithms are datadependent. Moreover, clustering results obtained by a particular algorithm might be changed due to some variations in the data set. However, one method could be more sensitive to variations in the data compared to any other method. For example, EFC algorithm is found to be more sensitive in this regard than the FCM algorithm. The reason behind this fact lies with the working principles of these two algorithms. Clusters are obtained through a single pass of the EFC algorithm, whereas FCM is an iterative algorithm. However, the changes in the clusters are found to be nominal. The basic characteristic identity of the clusters will remain the same.

\section{Concluding remarks}

The primary aim of the present study is to investigate whether a clustering of mechanisms based on quantitative analysis is feasible. To the best of the authors' knowledge, it is the first attempt to cluster the mechanisms. Two algorithms, namely EFC and FCM have been utilized for clustering of the mechanisms. Both the algorithms are able to do the clustering efficiently and a common trend of the clusters has been obtained. However, a more distinct set of clusters has been obtained by the EFC algorithm, whereas the FCM algorithm has yielded a set of more compact clusters. It has happened due to inherent properties of these two clustering algorithms. The obtained multidimensional clusters have been mapped into 2-D for visualization using a topology preserving tool like SOM. These 2-D clusters of mechanisms will help the designer or practical engineer to choose an appropriate mechanism to solve a particular problem. However, he will have to go back to multi-dimensional information of the selected mechanism to carry out further analysis of the same. Thus, this study helps to develop a quantifiable procedure for type synthesis of the mechanisms. 


\section{Scope for future study}

Ideally, the clusters should be distinct and compact too. To ensure this, a new clustering algorithm may be proposed, in which the cluster centers will be selected initially using the EFC algorithm from a set of data points to be clustered and then, they will be locally (within the cluster) updated to ensure compactness of the clusters using the FCM algorithm. An early version of this new proposal of clustering algorithm has recently been reported by Dey et al (2011), but there is a chance of further improvement of its performance. It will be studied in future. The present work may help the engineers to carry out type synthesis of the mechanisms, which can also be extended further, in future, to develop an expert system for the same. Thus, this study can be considered as one of the important steps for developing an expert system of the mechanisms. The expert system could be helpful to select an appropriate mechanism to serve a specific purpose. It has been kept in the scope for future study. An extensive literature is available on various fuzzy clustering algorithms (Pratihar 2008). Clustering is done based on the concept of similarity among data points, which is again dependent on the distance measures. Both the distance and similarity can be expressed in different ways. Besides the FCM and EFC algorithms, some other fuzzy clustering techniques, namely fuzzy ISODATA (Dunn 1973), fuzzy K-nearest neighbourhood algorithm (Keller et al 1985) may be tried in future to solve the same problem. Modified versions of FCM (Wu \& Li 2008; Thomas et al 2009) and EFC (Chattopadhyay et al 2006) algorithms are also in use. Moreover, a comparative study between the FCM and hard Cmeans clustering algorithms may be carried out to tackle this problem. A few more clustering algorithms, namely CLARANS (Clustering Large Applications based on RANdomized Search) (Ng \& Han 1994), DBSCAN (Density-Based Spatial Clustering of Applications with Noise) (Ester et al 1996) have also been reported for handling large spatial data sets. The present study may be extended in future using these clustering algorithms also.

\section{Acknowledgements}

The authors thank the Alexander von Humboldt Foundation, Germany, for the financial support, which made this investigation possible. They are grateful to the group of experts working in the field of mechanisms, who have helped to collect the data related to membership values of the mechanisms with respect to different attributes.

Appendix A. A list of 80 mechanisms considered in the present study.

\begin{tabular}{lll}
\hline No. & \multicolumn{1}{c}{ Reference } & \multicolumn{1}{c}{ Name of the mechanism } \\
\hline 1 & IGM 102b in Dittrich et al $(1993)$ & Crank rocker mechanism \\
2 & IGM 103b in Dittrich et al $(1993)$ & Slider crank mechanism \\
3 & IGM 103c in Dittrich et al $(1993)$ & Scotch yoke mechanism \\
4 & IGM 104b in Dittrich et al $(1993)$ & Oscillating cylinder mechanism \\
5 & IGM 202 in Dittrich et al $(1993)$ & Coupler curve dwell mechanism \\
6 & IGM 203/205 in Dittrich et al $(1993)$ & Coupler curve dwell mechanism \\
7 & IGM 204 in Dittrich et al $(1993)$ & Oscillating dwell mechanism \\
8 & IGM 208 in Dittrich et al $(1993)$ & Continuous rotary dwell mechanism \\
9 & IGM 216 in Dittrich et al $(1993)$ & Geared n-bar dwell mechanism \\
10 & IGM 217 in Dittrich et al $(1993)$ & \\
\hline
\end{tabular}


Appendix A. contd.

\begin{tabular}{|c|c|c|}
\hline No. & Reference & Name of the mechanism \\
\hline 11 & IGM 221 in Dittrich et al (1993) & Continuous rotary dwell mechanism \\
\hline 12 & IGM 232 in Dittrich et al (1993) & Mechanism with constant velocity \\
\hline 13 & IGM 250/253 in Dittrich et al (1993) & Spherical non-displaced slider crank \\
\hline 14 & IGM 254 in Dittrich et al (1993) & Spherical Geneva mechanism (external) \\
\hline 15 & IGM 264/265 in Dittrich et al (1993) & Spherical continuous rotary dwell mechanism \\
\hline 16 & IGM 307 in Dittrich et al (1993) & Geneva mechanism (external) \\
\hline 17 & IGM 308 in Dittrich et al (1993) & Geneva mechanism (internal) \\
\hline 18 & IGM 311 in Dittrich et al (1993) & Deep drawing press \\
\hline 19 & IGM 312 in Dittrich et al (1993) & Elliptic gear train \\
\hline 20 & IGM 601 in Dittrich et al (1993) & Plough blade guiding mechanism \\
\hline 21 & IGM 602 in Dittrich et al (1993) & Mechanism for opening an engine bonnet \\
\hline 22 & IGM 603 in Dittrich et al (1993) & Garage door guiding mechanism without roller rails \\
\hline 23 & IGM 608 in Dittrich et al (1993) & Parallel closing gripper \\
\hline 24 & IGM 610 in Dittrich et al (1993) & Straight line mechanism (Watt/Evans) \\
\hline 25 & IGM 612 in Dittrich et al (1993) & Crank rocker straight line mechanism \\
\hline 26 & KH 1.1.1.11 in Hain (1973) & $\begin{array}{l}\text { Six-bar linkage for oscillating rocking motion } \\
\text { with regard to optimum transmission angle }\end{array}$ \\
\hline 27 & KH 1.1.1.12 in Hain (1973) & Six-bar power-lift \\
\hline 28 & KH 1.1.2.1 in Hain (1973) & Eccentric type crank rocker \\
\hline 29 & KH 1.1.2.7 in Hain (1973) & $\begin{array}{l}\text { Double crank with a down-streamed crank rocker } \\
\text { for quick forward and slow return motion }\end{array}$ \\
\hline 30 & KH 1.1.2.13 in Hain (1973) & $\begin{array}{l}\text { Six-bar coupler mechanism, output slider } \\
\text { with constant velocity and quick return }\end{array}$ \\
\hline 31 & KH 2.1.1 in Hain (1973) & $\begin{array}{l}\text { Double crank with a down-streamed double crank } \\
\text { to increase the degree of irregularity }\end{array}$ \\
\hline 32 & KH 2.2.2 in Hain (1973) & $\begin{array}{l}\text { Equivalent linkage for a parallel crank mechanism, } \\
\text { containing rolls }\end{array}$ \\
\hline 33 & KH 3.3.2/3.3 in Hain (1973) & Double chain gear with higher cycloids \\
\hline 34 & KH 4.1.1 in Hain (1973) & Straight line guidance by four-bar linkage \\
\hline 35 & KH 5.1.3 in Hain (1973) & Linkage with two frame joints \\
\hline 36 & KH 5.1.4 in Hain (1973) & Eight-bar dwell mechanism \\
\hline 37 & KH 5.2.1 in Hain (1973) & Cam mechanism with roller lever as output link \\
\hline 38 & KH 5.2.3 in Hain (1973) & Cam mechanism with cam as output link \\
\hline 39 & KH 5.2.4 in Hain (1973) & Yoke cam for oscillating motion \\
\hline 40 & KH 7.1 in Hain (1973) & $\begin{array}{l}\text { Spatial linkage for transforming rotary motion } \\
\text { to turning and sliding motion }\end{array}$ \\
\hline 41 & KH 8.2.2 in Hain (1973) & Six-bar linkage with variation of the coupler curves \\
\hline 42 & KH 10.1.2 in Hain (1973) & $\begin{array}{l}\text { Six-bar dwell mechanism with rectilinear } \\
\text { translation, mixer-settler }\end{array}$ \\
\hline 43 & KH 10.1.4 in Hain (1973) & $\begin{array}{l}\text { Six-bar linkage with parallel coupler motion } \\
\text { for tape skip }\end{array}$ \\
\hline 44 & KH 10.1.5 in Hain (1973) & End cutting nippers with lever-assisted joint \\
\hline 45 & KH 10.3.2 in Hain (1973) & $\begin{array}{l}\text { Six-bar dwell linkage with rectilinear translation, } \\
\text { hauling gear }\end{array}$ \\
\hline
\end{tabular}


Appendix A. contd.

\begin{tabular}{|c|c|c|}
\hline No. & Reference & Name of the mechanism \\
\hline 46 & KH 10.3.4 in Hain (1973) & Front- and rear-loader on farming tractor \\
\hline 47 & KH 10.4.6 in Hain (1973) & $\begin{array}{l}\text { Six-bar dwell mechanism with oscillating } \\
\text { rectilinear coupler motion }\end{array}$ \\
\hline 48 & 1.1.2.1.2 P3 No.2 in Bock (1980) & Crank rocker with highly flattened coupler curve \\
\hline 49 & 1.1.2.1.3 P15 No.1 in Bock (1980) & Peaucellier mechanism \\
\hline 50 & 1.1.2.2. P3 No.2 in Bock (1980) & Six-bar scissors mechanism \\
\hline 51 & 1.4 P5 No.3 in Bock (1980) & Planetary gear train (workpart handling) \\
\hline 52 & 2.1.1.1 P2 No.1 in Bock (1980) & Epicyclic gear train \\
\hline 53 & 2.1.1.1 P8 No.4 in Bock (1980) & Oldham's coupling \\
\hline 54 & 2.1.1.3 P4 No.4 in Bock (1980) & Worm gear \\
\hline 55 & 2.3.1.1 P4 No.1 in Bock (1980) & Cam mechanism with toothed wheel as output link \\
\hline 56 & 2.3.1.2 P1a No.1 in Bock (1980) & Toothed gearing with two eccentric gears \\
\hline 57 & 2.3.1.3 P1 No.4 in Bock (1980) & Hooke's joint/universal joint \\
\hline 58 & 2.5.1 P1 No.4 in Bock (1980) & Ratchet mechanism \\
\hline 59 & 2.5.1 P4 No.1 in Bock (1980) & Cam-rocking follower mechanism \\
\hline 60 & 2.5.3.7 P1 No.1 in Bock (1980) & $\begin{array}{l}\text { Cylindrical cam mechanism with groove, } \\
\text { step-mechanism with rotary output }\end{array}$ \\
\hline 61 & 2.6.4 P1 No.1 in Bock (1980) & $\begin{array}{l}\text { Cylindrical cam mechanism, step-mechanism } \\
\text { with linear output }\end{array}$ \\
\hline 62 & 2.6.6 P3 No.1 in Bock (1980) & Elevating platform with step motion \\
\hline 63 & 2.7.1.1.1 P2 No.1 in Bock (1980) & Inverted slider crank (slotted lever quick return mechanism) \\
\hline 64 & 2.7.2.1.1.8 P1 No.3 in Bock (1980) & Coupler mechanism with screw wheel as input link \\
\hline 65 & 2.8.1.1.1.1 P2 No.1 in Bock (1980) & Inverted slider crank with coupling link, slider as output link \\
\hline 66 & 2.8.1.1.1.2 P1 No.1 in Bock (1980) & Cam-translating follower (roller) \\
\hline 67 & 9.0/1 in Dittrich \& Braune (1987) & Cam-translating follower (flat-face) \\
\hline 68 & 7.33 in Vomer (1992) & Harmonic drive \\
\hline 69 & 7.32 in Vomer (1992) & Cyclo-drive \\
\hline 70 & 8.1/2 in Dittrich \& Braune (1987) & Cone pulley variable speed drive \\
\hline 71 & 10.11-4 in Mallik et al (1994) & Multiple speed gear box \\
\hline 72 & Artobolevsky (1977) & Mini-max variable speed drive \\
\hline 73 & & Rack \& Pinion mechanism \\
\hline 74 & & Clutch mechanism \\
\hline 75 & 4.3 in Mallik et al (1994) & Six-link wiper mechanism \\
\hline 76 & & Sprocket and chain drive \\
\hline 77 & Artobolevsky (1977) & Automatic focusing mechanism \\
\hline 78 & & Belt-step pulley drive \\
\hline 79 & Artobolevsky (1977) & Variable speed ball drive \\
\hline 80 & 4.2-4 in Mallik et al (1994) & Pantograph copying mechanism \\
\hline
\end{tabular}




\begin{tabular}{|c|c|c|c|c|c|c|c|c|c|c|c|c|c|}
\hline 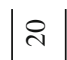 & $\stackrel{?}{0}$ & $\stackrel{?}{0}$ & $\begin{array}{ll}0 & 0 \\
0 & 0 \\
0 & 0 \\
0\end{array}$ & $\begin{array}{ll}\infty & 0 \\
0 & 0 \\
0 & 0\end{array}$ & 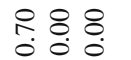 & $\stackrel{8}{0}$ & $\stackrel{8}{\circ}$ & $\stackrel{8}{0}$ & $\underset{-}{8}$ & 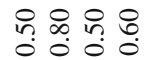 & $\stackrel{8}{0}$ & n̊? & $\stackrel{8}{\circ}$ \\
\hline 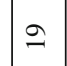 & $\stackrel{8}{\circ}$ & $\stackrel{8}{\circ}$ & $\begin{array}{ll}\infty & 0 \\
\infty & n \\
0 & 0\end{array}$ & $\begin{array}{l}8: \\
0 \\
0\end{array}$ & : 8.8 & $\stackrel{8}{8}$ & $\stackrel{8}{8}$ & $\stackrel{8}{\circ}$ & 8 & 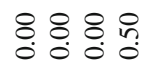 & กิ & $\stackrel{8}{0}$ & $\stackrel{8}{8}$ \\
\hline$\stackrel{\infty}{-}$ & $\stackrel{R}{0}$ & \&. & $\begin{array}{ll}\infty & 0 \\
0 & \infty \\
0 & 0\end{array}$ & $\begin{array}{ll}\infty & 0 \\
\infty & \infty \\
0 & 0 \\
0\end{array}$ & $\begin{array}{l}\circ \\
\stackrel{0}{0} \\
\circ\end{array}$ & $\stackrel{8}{8}$ & $\underset{8}{8}$ & $\stackrel{8}{-}$ & $\stackrel{8}{0}$ & 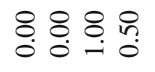 & $\stackrel{8}{0}$ & ?̊n & $\stackrel{8}{0}$ \\
\hline$=$ & ़ֻ! & $\stackrel{?}{+}$ & 융ำ & $\stackrel{0}{\circ} \stackrel{9}{0}$ & \& \& & $\stackrel{8}{\circ}$ & $\stackrel{8}{\circ}$ & $\stackrel{8}{\circ}$ & $\stackrel{8}{\circ}$ & 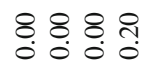 & $\underset{8}{8}$ & $\stackrel{8}{\circ}$ & $\stackrel{8}{\circ}$ \\
\hline$\stackrel{0}{2}$ & $\stackrel{0}{+}$ & $\stackrel{q}{+}$ & 눙 웅 & 유? & $\underset{0}{8} 8.8$ & 8 & $\stackrel{8}{8}$ & 8 & $\stackrel{8}{0}$ & $\begin{array}{l}8: 8 \% \\
0.09 \\
0.0\end{array}$ & $\stackrel{8}{8}$ & $\stackrel{8}{0}$ & 8 \\
\hline$\cong$ & $\stackrel{q}{9}$ & q & $\begin{array}{l}8 \\
0\end{array}$ & ల్లి & \begin{tabular}{l}
8 \\
\hdashline \\
0
\end{tabular} & 8 & $\stackrel{8}{8}$ & 8 & $\stackrel{8}{0}$ & \begin{tabular}{l}
$8: 8$ \\
\hdashline
\end{tabular}$\stackrel{8}{\circ} \stackrel{0}{\circ}$ & $\stackrel{8}{\circ}$ & $\stackrel{8}{\circ}$ & $\stackrel{8}{\circ}$ \\
\hline \pm & $\stackrel{+}{+}$ & ़ֻ? & in & $\begin{array}{l}0 \\
0 \\
0\end{array}$ & 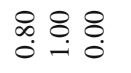 & $\stackrel{8}{8}$ & $\stackrel{8}{-}$ & $\stackrel{8}{\circ}$ & $\stackrel{8}{0}$ & $\begin{array}{llll}8 & 8 & 8 & 0 \\
0 & 0 & 0 & 0\end{array}$ & $\stackrel{8}{\circ}$ & $\stackrel{8}{8}$ & $\stackrel{8}{\circ}$ \\
\hline 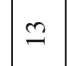 & $\stackrel{o}{+}$ & $\stackrel{q}{+}$ & in & 웅 & $\stackrel{2}{2} 8$. & $\underset{8}{8}$ & 8 & 8 & 8 & $\begin{array}{l}8: 0 \\
0 \\
0\end{array}$ & 8 & 8 & 8 \\
\hline$\simeq$ & $\stackrel{8}{:}$ & $\stackrel{n}{n}$ & 유ำ & तิ तิ & 용 & $\stackrel{8}{8}$ & $\stackrel{8}{\circ}$ & $\stackrel{8}{\circ}$ & $\stackrel{8}{\circ}$ & 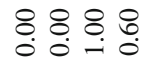 & $\stackrel{8}{\circ}$ & तั & $\stackrel{8}{8}$ \\
\hline$=$ & $\stackrel{?}{9}$ & @ి & $\begin{array}{l}0 \\
\stackrel{1}{0} \\
0\end{array}$ & ले గి & 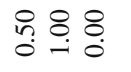 & $\stackrel{8}{0}$ & $\stackrel{8}{\circ}$ & $\stackrel{8}{\circ}$ & $\stackrel{8}{0}$ & \begin{tabular}{lll}
8 & 8 & 0 \\
\hdashline & 0 & 0 \\
0 & 0 & 0
\end{tabular} & ?̊. & $\stackrel{8}{0}$ & $\stackrel{8}{8}$ \\
\hline 은 & 웅 & ֻి & $\stackrel{8}{: 0}:$ & సิ તิ & 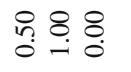 & $\underset{8}{8}$ & $\underset{0}{8}$ & $\stackrel{8}{-}$ & $\stackrel{8}{0}$ & 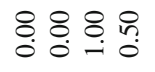 & $\stackrel{?}{0}$ & @ి & $\stackrel{8}{\circ}$ \\
\hline$a$ & तิ & @ి & กิ กิ & సิ ণิ స్ & : & 8 & $\stackrel{8}{-}$ & $\stackrel{8}{\circ}$ & $\stackrel{8}{0}$ & 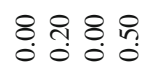 & $\stackrel{8}{0}$ & $\stackrel{8}{0}$ & $\stackrel{8}{0}$ \\
\hline$\infty$ & $\stackrel{8}{0}$ & $\stackrel{n}{2}$ & $\begin{array}{l}8 \\
8 \\
0\end{array}$ & $\begin{array}{l}\Re \\
0 \\
0\end{array}$ & 우 8 : 8 & $\stackrel{8}{-}$ & $\stackrel{8}{\circ}$ & 8 & $\stackrel{8}{0}$ & 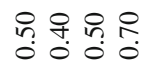 & $\stackrel{2}{0}$ & $\stackrel{n}{\circ}$ & $\stackrel{8}{\circ}$ \\
\hline$r$ & in & $\stackrel{n}{n}$ & 유ํ तิ & 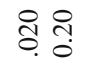 & $\begin{array}{l}8 \\
8 \\
0\end{array}$ & $\stackrel{8}{8}$ & $\stackrel{8}{0}$ & $\stackrel{8}{\circ}$ & $\stackrel{8}{0}$ & 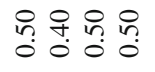 & $\stackrel{?}{0}$ & $\stackrel{n}{n}$ & $\stackrel{8}{8}$ \\
\hline 6 & $\begin{array}{l}8 \\
:\end{array}$ & $\stackrel{n}{i}$ & $\begin{array}{l}8 \\
\& \\
0 \\
0\end{array}$ & 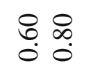 & 웅 & $\stackrel{8}{0}$ & $\stackrel{8}{0}$ & 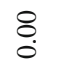 & 8 & 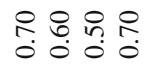 & $\stackrel{n}{0}$ & లి & $\stackrel{8}{0}$ \\
\hline$n$ & ?̊ & $\stackrel{n}{n}$ & 웅 ֻ & సે సે & 요 & $\stackrel{8}{8}$ & $\stackrel{8}{0}$ & $\stackrel{8}{\circ}$ & $\stackrel{8}{\circ}$ & $\begin{array}{ccc}0 & 0 & 0 \\
0 & 0 & 0 \\
0 & 0 & 0 \\
0\end{array}$ & $\stackrel{?}{\circ}$ & ڤి. & $\stackrel{8}{\circ}$ \\
\hline$\nabla$ & 옹 & $\stackrel{?}{0}$ & 웅 & 웅 & $\begin{array}{l}8 \\
8 \\
0\end{array}$ & $\stackrel{8}{8}$ & $\stackrel{8}{0}$ & $\stackrel{8}{8}$ & $\stackrel{8}{0}$ & 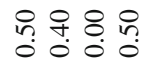 & $\stackrel{8}{\circ}$ & $\stackrel{n}{n}$ & $\stackrel{8}{8}$ \\
\hline$m$ & $\stackrel{0}{+}$ & ?̊ & ํㅠำ & 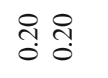 & : $8: 8$ & $\stackrel{8}{8}$ & 8 & $\stackrel{8}{\circ}$ & 8 & 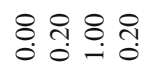 & $\stackrel{8}{0}$ & $\stackrel{8}{\circ}$ & $\stackrel{8}{\circ}$ \\
\hline$N$ & ڤn & $\stackrel{n}{\mathfrak{0}}$ & 윰 & 웅 & 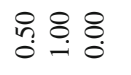 & $\underset{8}{8}$ & $\stackrel{8}{\circ}$ & $\stackrel{8}{-}$ & $\stackrel{8}{0}$ & 유요 & $\stackrel{0}{0}$ & in & 8 \\
\hline- & $\stackrel{8}{0}$ & $\stackrel{n}{a}$ & $\begin{array}{l}8 \\
0 \\
0\end{array}$ & $\begin{array}{l}R \\
\stackrel{\infty}{0} \\
0\end{array}$ & 웅 8 : 8 & $\underset{8}{8}$ & $\stackrel{8}{0}$ & $\stackrel{8}{\circ}$ & $\stackrel{8}{0}$ & 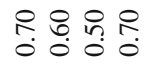 & $\stackrel{8}{0}$ & $\stackrel{n}{0}$ & $\stackrel{8}{0}$ \\
\hline 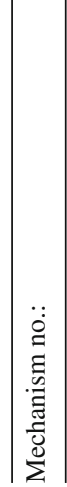 & 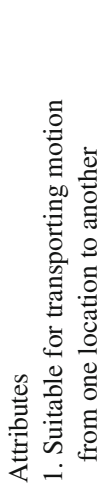 & 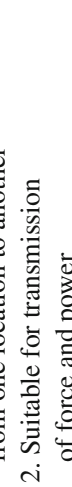 & 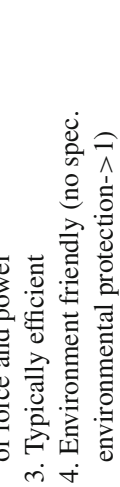 & 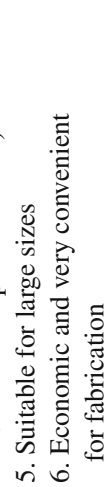 & 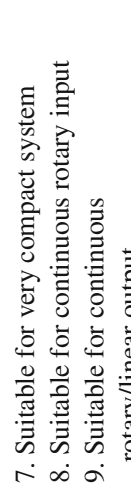 & 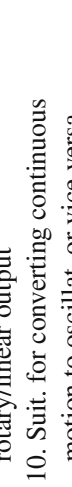 & 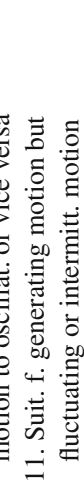 & 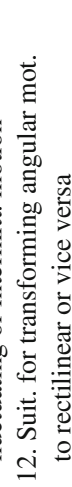 & 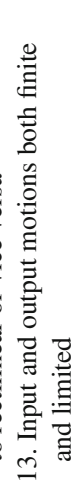 & 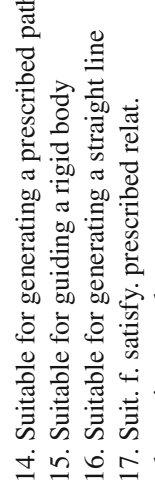 & 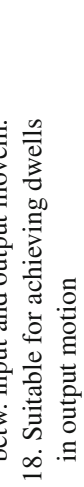 & 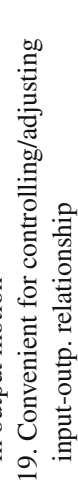 & 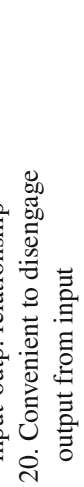 \\
\hline
\end{tabular}




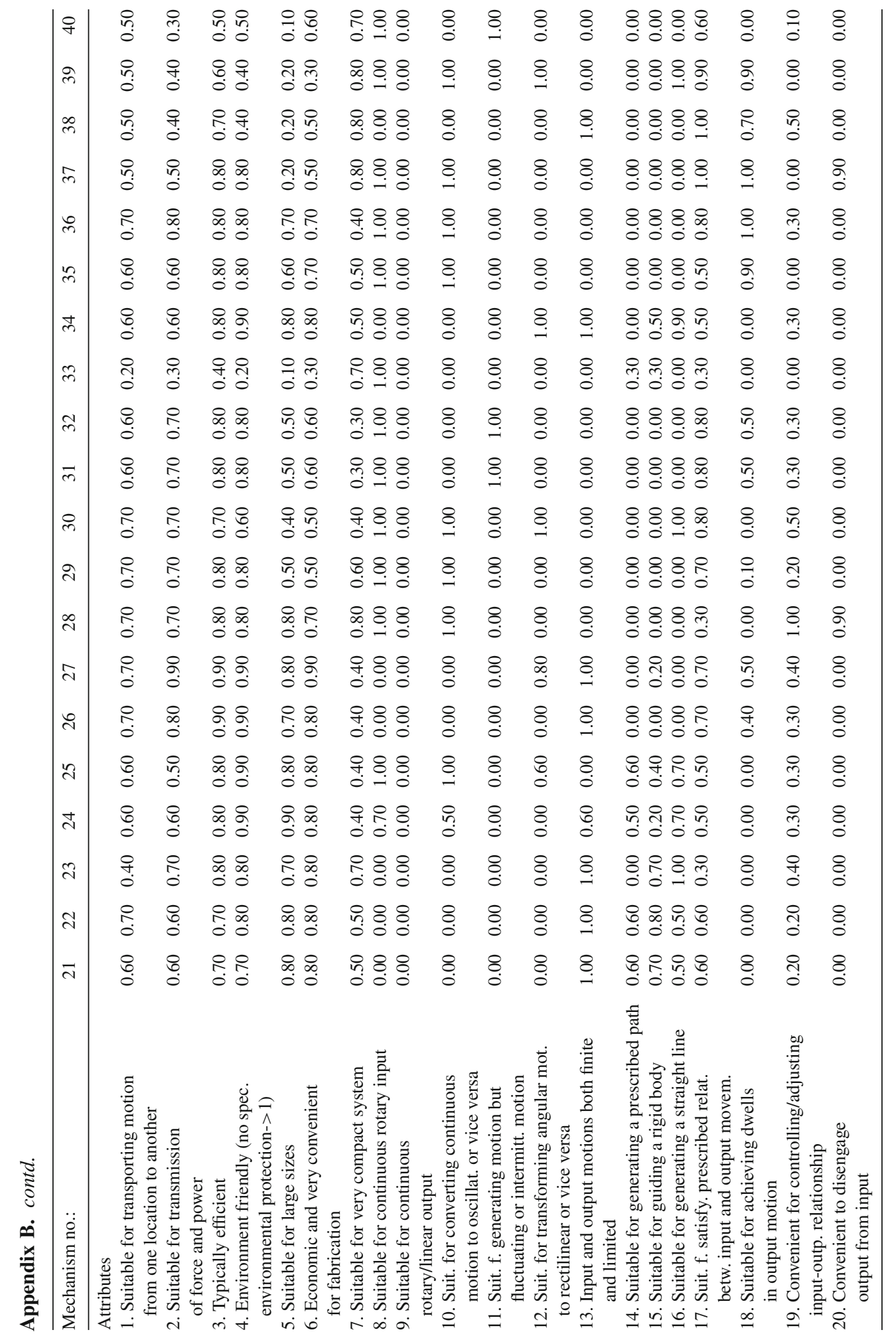




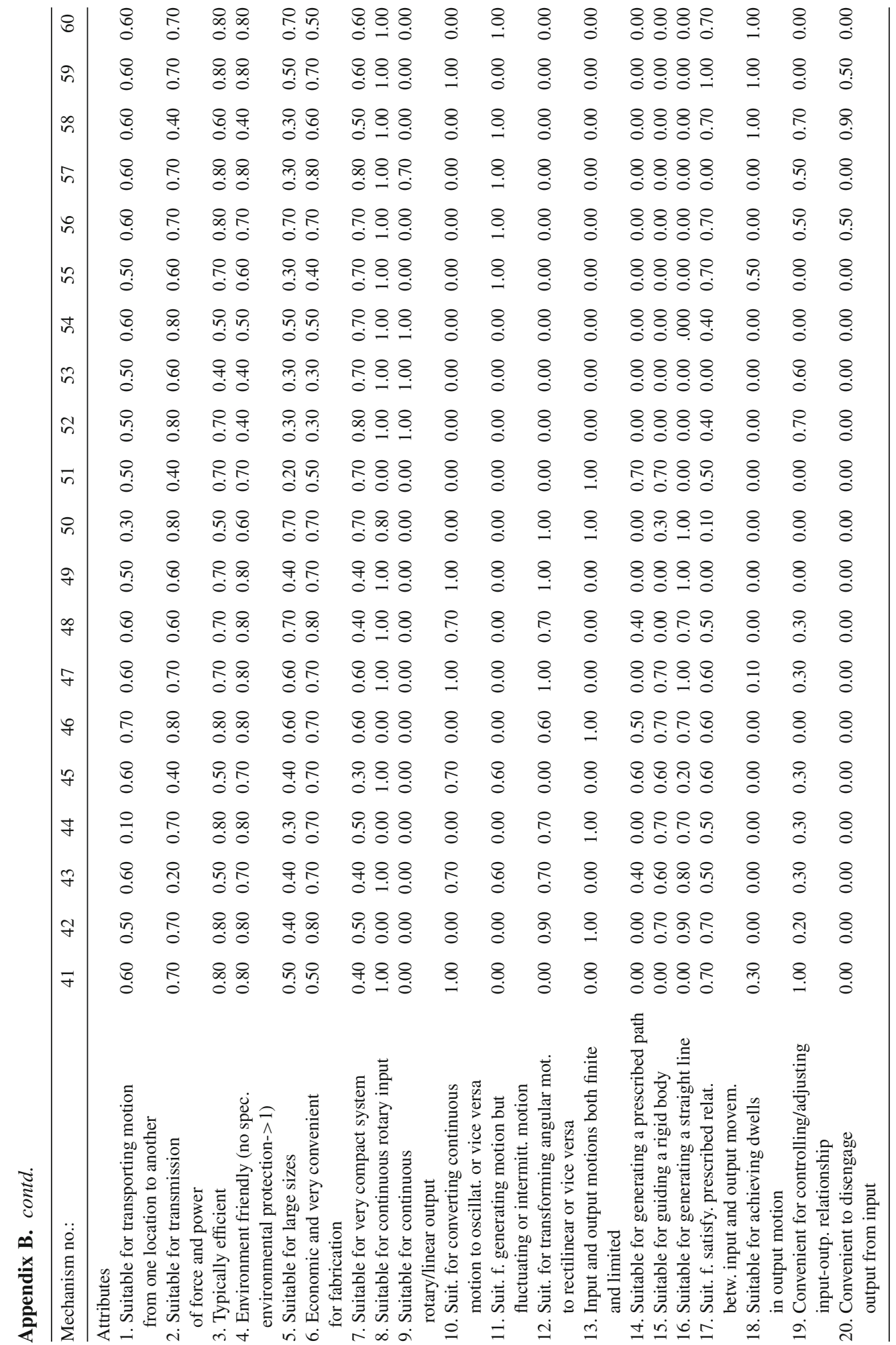




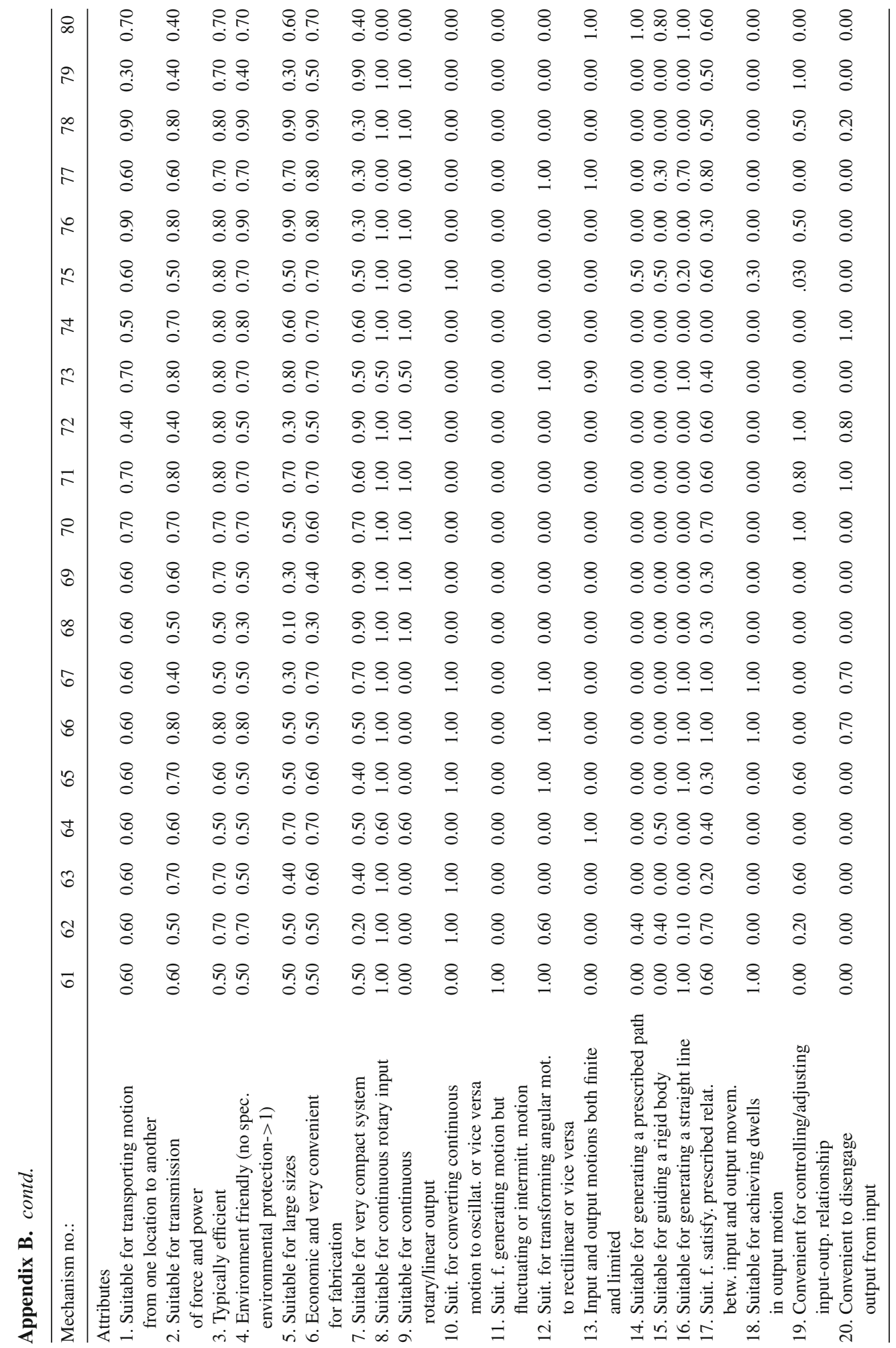




\section{References}

Artobolevsky I I 1977 Mechanisms in modern engineering design: A hand-book for engineers, designers and inventors, Moscow: Mir Publishers, vol. IV

Bezdek J C 1973 Fuzzy mathematics in pattern classification, Ph.D. thesis, Applied Mathematics Center, Cornell University, Ithaca

Bock A 1980 Arbeitsbatter fur die Konstruktion von Mechanismem. Kammer der Technik, BV Suhl Bereich Wissenschaft und Technik, Suhl

Chattopadhyay S, Pratihar D K and De Sarkar S C 2006 Performance studies of some similarity-based fuzzy clustering algorithms, Int. J. Performability Eng. 2(2): 191-200

Dey V, Pratihar D K and Datta G L 2011 Genetic algorithm-tuned entropy-based fuzzy C-means algorithm for obtaining distinct and compact clusters, Fuzzy Optimization and Decision Making, 10: 153-166

Dittrich G and Braune R 1987 Getriebetechnik in Beispielen. Munchen: Oldenbourg Verlag

Dittrich G, Muller J and Wehn V 1993 IGM-Getriebesammlung. Selbstverlag. Loseblatt-Ausgabe, 2. Lieferung, Stand Dezember 1993, Aachen

Dunn J C 1973 A fuzzy relative of the ISODATA process and its use in detecting compact well-separated clusters, J. Cybernetics 3: 32-57

Ester M, Kriegel H P, Sander J and Xu X 1996 A density-based algorithm for discovering clusters in large spatial databases with noise, Proc. of 2nd International conference on knowledge discovery and data mining (KDD), Portland, Oregon, pp. 226-231

Goldberg D E 1989 Genetic algorithms in search, optimization and machine learning, Reading, MA: USA, Addison-Wesley

Hain K 1973 Getriebebeispiel-Atlas. Dusseldorf: VDI-Verlag GmbH

Holland J H 1975 Adaptation in natural and artificial systems. Ann Arbor, MI, USA: The University of Michigan Press

Keller J, Gary M R and Givens J A 1985 A fuzzy K-nearest neighbor algorithm, IEEE Trans. On Systems, Man and Cybernetics, SMC-15, 4, pp. 580-585

Kohonen T 1995 Self-Organizing Maps, Heidelberg, Germany: Springer-Verlag

Mallik A K, Ghosh A and Dittrich G 1994 Kinematic analysis and synthesis of mechanisms, Boca Raton: CRC Press

Ng R T and Han J 1994 Efficient and effective clustering method for spatial data mining, Proc. of 20th International Conference on very large data bases, Santiago, Chile, pp. 144-155

Pratihar D K 2008 Soft Computing, New Delhi: Narosa Publishing House

Thomas B, Raju G and Wangmo S 2009 A modified fuzzy c-means algorithm for natural data exploration, World Academy of Science, Engineering and Technology, 49: 478-481

Vomer J 1992 Getriebetechnik Grundlagen. Berlin: Verlag Technik

Wang H P and Li J-K 1991 Computer-aided process planning (Chapter 6). Elsevier Science, Amsterdam

Wu J and Li T 2008 A modified fuzzy c-means algorithm for collaborative filtering, Proc. of 2nd NetflixKDD Workshop, Las Vegas, USA

Yao J, Dash M, Tan S T and Liu H 2000 Entropy-based fuzzy clustering and fuzzy modeling, Fuzzy Sets and Systems, 113: 381-388

Zadeh L A 1965 Fuzzy sets, Information and Control, 8: 338-353 